Special issue of the International Conference on Computational and Experimental Science and Engineering (ICCESEN 2014)

\title{
Simulation of Carbon Ions Interactions with Monocrystalline Silicon Targets
}

\author{
A. Bouguerra And R. LABBAni*
}

Laboratoire de Physique Mathématique et Subatomique, Département de Physique, Faculté des Sciences Exactes, Université Constantine 1, Route de Ain El Bey, 25000 Constantine, Algeria

\begin{abstract}
In this work, several phenomena related to carbon ion implantation into $\mathrm{Si}(100)$ targets were simulated. The investigation was performed using Crystal-TRIM code (crystal-transport and range of ions in matter) under different conditions. In particular, we simulated the carbon profiles with respect to: (i) ions beam (energy, dose, orientation); (ii) substrate (temperature, crystallographic orientation). Two particular cases were taken into account: (i) implantation of $80 \mathrm{keV} \mathrm{C} \mathrm{C}^{+}$to a fluence of $2.7 \times 10^{17} \mathrm{ion} / \mathrm{cm}^{2}$ at room temperature; (ii) implantation of $40 \mathrm{keV} \mathrm{C} \mathrm{C}^{+}$to a fluence of $6.5 \times 10^{17} \mathrm{ion} / \mathrm{cm}^{2}$ at substrate temperature of $400{ }^{\circ} \mathrm{C}$. For both cases, we used a tilt angle of $7^{\circ}$. Several results were obtained and compared with the Rutherford backscattering spectroscopy and elastic recoil detection analysis results provided by literature.
\end{abstract}

DOI: 10.12693/APhysPolA.128.B-67

PACS: 78.20.Bh, 85.40.Ry

\section{Introduction}

Carbon ion implantation into silicon wafers is one of interest preparation techniques of silicon carbide which is a promising material in electronic industry. This is due to its important properties: high thermal conductivity, wide band gap, and high breakdown electric field. Consequently, it is highly attractive for devices working at high-temperature, high power, and high resistance [1].

Crystal-TRIM code is an interesting tool to study ionmaterial interactions [2]. It is especially designed to study ion implantation into monocrystalline substrates of $\mathrm{Si}, \mathrm{Ge}$, or diamond. It is able to predict various phenomena such as: depth profiles of ions and damage in targets, nuclear and electronic energy deposition and channelling phenomena of ions in case of different crystallographic directions.

\section{Theory of Crystal-TRIM code}

\subsection{Assumptions}

Several assumptions are taken into account to study theoretically the binary collision in Crystal-TRIM code. It is based on several assumptions [3]. In particular, (i) the target composition is supposed unchanged by ion implantation, (ii) the velocity of target atoms is equal to zero prior to the collision with projectile, (iii) the motion of recoiled target atoms is not considered.

\subsection{Inter-atomic potential}

In case of very small distances between the nuclei, the repulsive interaction is supposed to be essentially

*corresponding author; e-mail: labbanire@gmail.com
Coulombic. At greater distances, the repulsive potential $V(r)$ can be obtained by multiplying the Coulombic repulsion with a screening function $\varphi(r / a)[4]$ :

$$
V(r)=\frac{1}{4 \pi \varepsilon_{0}} \frac{Z_{1} Z_{2} e^{2}}{r} \varphi\left(\frac{r}{a}\right),
$$

where $r$ is the interaction distance and $a$ is computed by

$$
a=\frac{0.8854 a_{0}}{Z_{1}^{0.23}+Z_{2}^{0.23}} .
$$

$Z_{1}, Z_{2}$ are the atomic numbers of the projectile and the target, respectively. The quantity $a$ is the universal screening length, $a_{0}$ is the Bohr atomic radius $\left(a_{0}=0.529 \AA\right)$ and $e$ is the charge of the electron $\left(e=1.6 \times 10^{-19} \mathrm{C}\right)$. We note that the universal potential $V(r)$ is reported to be a precise approximation for many projectile target cases [4].

\subsection{Damage accumulation}

If $T_{n}$ is the transferred energy from the projectile to the primary-knock-on atom, this quantity is further transferred to secondary recoils by collision cascade. In the collision projectile target atom, the nuclear energy deposition is estimated by [3]:

$$
E_{n}=\frac{T_{n}}{1+k_{\mathrm{d}} g\left(\varepsilon_{\mathrm{d}}\right)},
$$

where

$$
\begin{aligned}
& k_{\mathrm{d}}=0.1334\left(Z_{2}^{2 / 3} / m_{2}^{1 / 2}\right), \\
& g\left(\varepsilon_{\mathrm{d}}\right)=\varepsilon_{\mathrm{d}}+0.4 \varepsilon_{\mathrm{d}}^{3 / 4}+3.4 \varepsilon_{\mathrm{d}}^{1 / 6}, \\
& \varepsilon_{\mathrm{d}}=0.01\left(T_{n} / Z_{2}^{\frac{7}{3}}\right) .
\end{aligned}
$$

$T_{n}$ and $m_{2}$ are the nuclear energy loss and target atom mass, respectively.

The electronic energy deposition is given by [3]:

$$
E_{\mathrm{el}}=\Delta E_{\mathrm{el}}+T_{n}-E_{n} \text {. }
$$


According to $E_{n}$ values, the number of displacements $N_{\mathrm{d}}$ is computed using the "modified" KinchinPease model [5]:

$$
\begin{aligned}
& N_{\mathrm{d}}=1 \text { if } E_{\mathrm{d}} \leq E_{n}<2.5 E_{\mathrm{d}}, \\
& N_{\mathrm{d}}=0.4\left(E_{n} / E_{\mathrm{d}}\right) \text { if } E_{n} \geq 2.5 E_{\mathrm{d}} .
\end{aligned}
$$

For instance, the displacement energy $E_{\mathrm{d}}$ used for silicon target is about $15 \mathrm{eV}$ [6].

For a depth interval $(x, x+\Delta x)$ of the silicon target, the probability $\mathrm{d} p_{\mathrm{d}}$ that a carbon pseudo-projectile displaces a target atom from its atomic site is given by [6]:

$$
\mathrm{d} p_{\mathrm{d}}=\frac{1}{n}\left(\frac{N_{\mathrm{d}}}{\Delta x} \frac{D_{0}}{N}\right)\left(1-p_{\mathrm{d}}\right),
$$

where $n$ is the atomic density of the target, $D_{0}$ is the implantation dose of $\mathrm{C}^{+}$and $N$ is the total number of pseudo-projectiles considered in the simulation.

By integration of Eq. (9), the probability of atomic displacement is estimated by [6]:

$$
p_{\mathrm{d}}=1-\exp \left(-\frac{1}{n} \frac{N_{\mathrm{d}}}{\Delta x} D_{0}^{\prime}\right),
$$

where $D_{0}^{\prime} \leq D_{0}[6]$. During ions implantation, various types of defects are created (vacancies, dislocations, and local amorphous regions).

\section{Results and discussion}

\subsection{Influence of ions beam parameters on carbon depth profiles}

In Fig. 1, we report the Crystal-TRIM simulation results obtained for different implantation energies. It is clear that carbon range profiles are Gaussian-like distribution which is logical since the simulation was run for a tilt of $7^{\circ}$. In other words, the target is seen as amorphous with respect to carbon ions beam. Also, we can note that the values of mean projected range $\left(R_{\mathrm{p}}\right)$ and straggling $\left(\Delta R_{\mathrm{p}}\right)$ increases and curves become deeper by increasing implantation energies. This is due to the fact that higher energies allow the ions beam to penetrate deeper in the substrate.

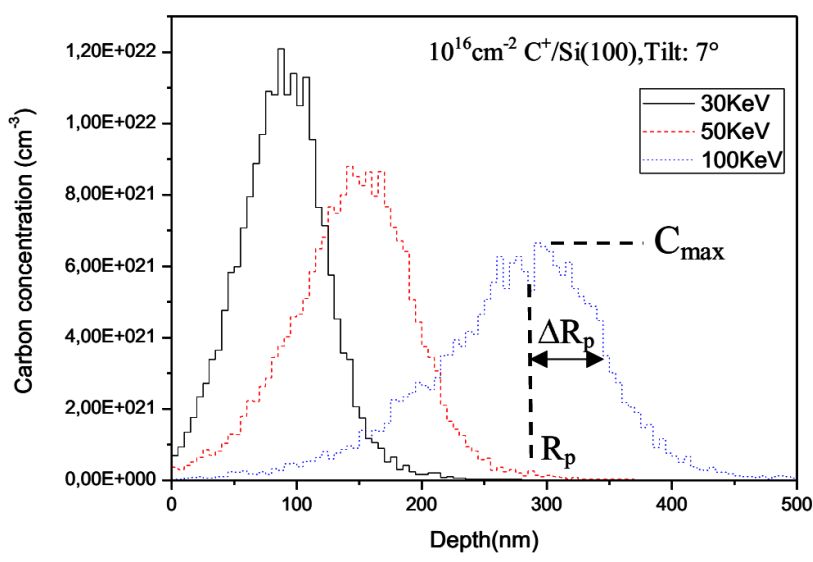

Fig. 1. Crystal-TRIM simulation results for depth profiles of carbon ions with different implantation energies.

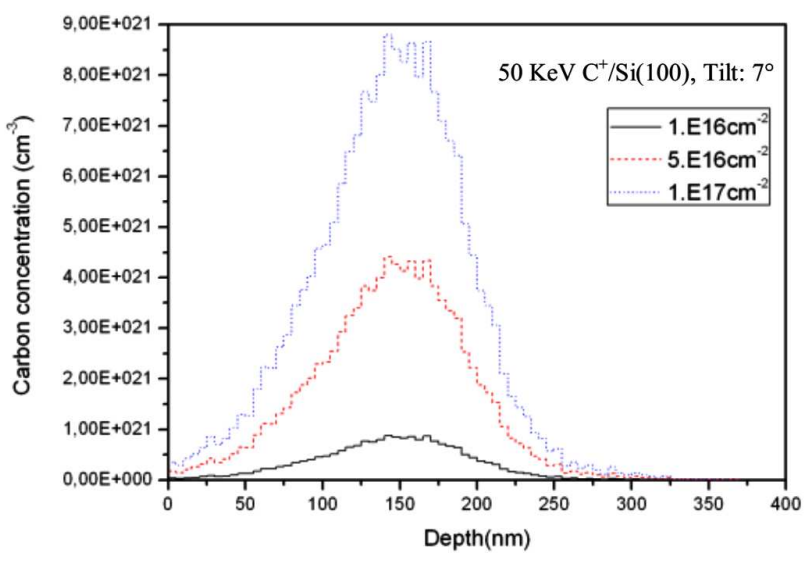

Fig. 2. Crystal-TRIM results corresponding to carbon depth profiles versus different implantation doses.

Concerning the effect of implantation dose, the simulation has also provided Gaussian-like distribution. Indeed, the variation of carbon range versus three different implantation doses $\left(1 \times 10^{16}, 5 \times 10^{16}\right.$ and $\left.1 \times 10^{17} \mathrm{~cm}^{-2}\right)$ is shown in Fig. 2. We note that the three curves have the same values of the projected range $\left(R_{\mathrm{p}}\right)$ and the standard deviation $\left(\Delta R_{\mathrm{p}}\right)$ (i.e. $R_{\mathrm{p}}=146 \mathrm{~nm}$ and $\Delta R_{\mathrm{p}}=50 \mathrm{~nm}$, respectively) but exhibit different values of $\mathrm{C}_{\max }^{+}$concentration. This is logical since different doses have been used.

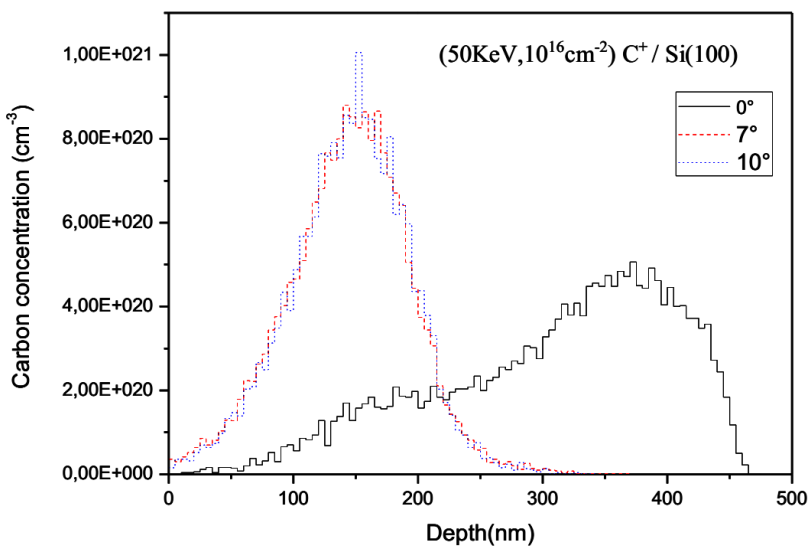

Fig. 3. Crystal-TRIM results coresponding to carbon depth profiles with different implantation tilt angles.

Concerning the tilt angle effect, Crystal-TRIM predictions have indicated a huge carbon atoms canalisation for the case of $0^{\circ}$. As shown in Fig. 3, the shape of the range profile for $0^{\circ}$ tilt is not a Gaussian-like distribution wich is due to channelling effect, whereas for $7^{\circ}$ and $10^{\circ}$ tilt, the curves are similar $\left(R_{\mathrm{p}}=147 \mathrm{~nm} ; \Delta R_{\mathrm{p}}=48 \mathrm{~nm}\right)$. Therefore it is recommended to avoid the $0^{\circ}$ tilt for homogeneous layers $\mathrm{SiC}$ elaboration.

\subsection{Influence of temperature and crystallographic orientation of substrate on carbon depth profiles}

Crystal-TRIM code has a greater advantage with regard to other codes because it takes into account the 
effects of temperature and crystallographic orientation of the substrate. In Fig. 4, three different substrate temperatures $\left(25^{\circ} \mathrm{C}, 200^{\circ} \mathrm{C}\right.$ and $\left.400^{\circ} \mathrm{C}\right)$ have been selected. The simulation was run for two implantation energies (50 keV and $80 \mathrm{keV}$ ) where we note that depth profiles distribution does not depend on the temperature of substrates. $R_{\mathrm{p}}$ is approximately equal to $145 \mathrm{~nm}$ and $222 \mathrm{~nm}$ for $50 \mathrm{keV}$ and $80 \mathrm{keV}$ energies, respectively, whereas the standard deviation is estimated to be about $48 \mathrm{~nm}$ and $62 \mathrm{~nm}$ for the same energies.

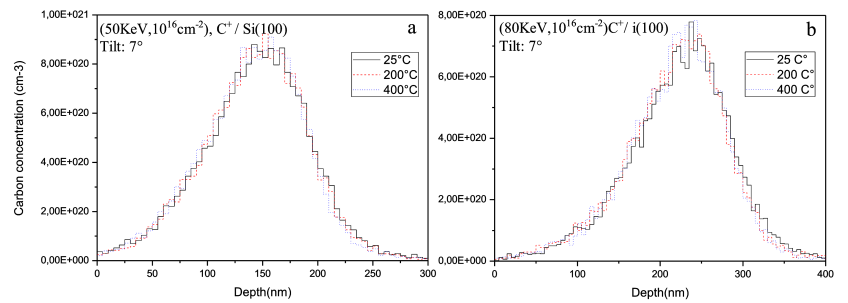

Fig. 4. Crystal-TRIM simulation results for carbon depth profiles, with different substrate temperatures, for $50 \mathrm{keV}$ (a) and $80 \mathrm{keV}$ (b) implantation energies.

The effect of substrate crystallographic orientation has also been studied in this work. In Fig. 5, $\langle 100\rangle$ and $\langle 111\rangle$ orientations have been selected because they are generally used for compound fabrication. As it can be seen, $\langle 100\rangle$ orientation yields slightly deeper carbon profile with respect to $\langle 111\rangle$. This is logical since along $\langle 100\rangle$ direction, less substrate atoms number is knocked-on with regard to $\langle 111\rangle$ case.

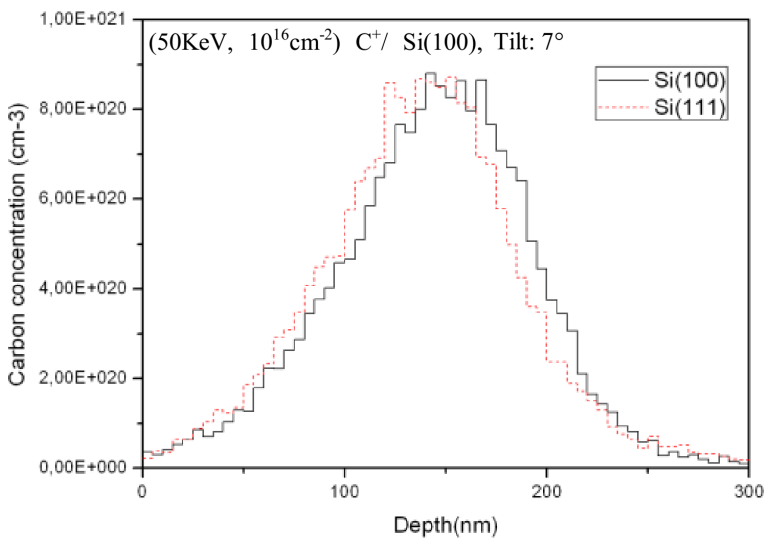

Fig. 5. Crystal-TRIM simulation results for depth profiles of carbon atoms implanted in Si (100) and Si (111) substrates.

\subsection{Simulation of damage profile}

As shown in Fig. 6, we note that the projected range of damage $R_{\mathrm{d}}$ (Fig. 6a) was lower than the projected range of carbon atoms $R_{\mathrm{p}}$ (Fig. 6b) which is in agreement with literature [7].
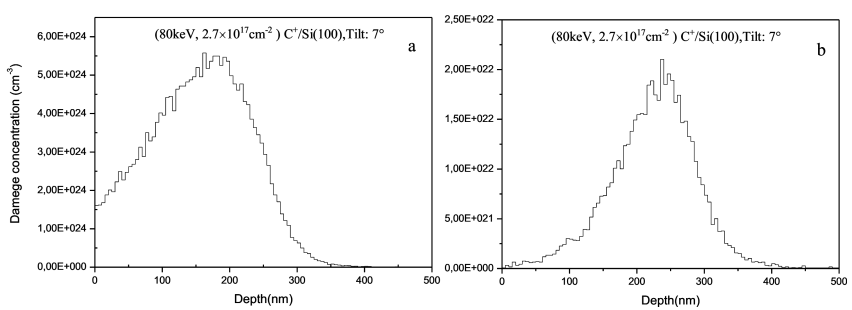

Fig. 6. Crystal-TRIM simulation results for damage concentration profile (a) and carbon atoms concentration profile (b).

\subsection{Comparison of experimental and theoretical results}

In this section, we have performed comparison between the simulated and experimental results which have been provided in literature [8]. Two particular cases have been selected: (i) implantation of $80 \mathrm{keV} \mathrm{C}^{+}$to a fluence of $2.7 \times 10^{17} \mathrm{ion} / \mathrm{cm}^{2}$ at room temperature, (ii) implantation of $40 \mathrm{keV} \mathrm{C} \mathrm{C}^{+}$to a fluence of $6.5 \times 10^{17} \mathrm{ion} / \mathrm{cm}^{2}$ at substrate temperature of $400^{\circ} \mathrm{C}$.

TABLE

Comparison of carbon depth profile parameters determined by Crystal-TRIM code (i.e. this work) with experimental ERDA and RBS data provided by literature [8].

\begin{tabular}{c|c|c|c}
\hline \hline Method & Depth $_{\max }[\mathrm{nm}]$ & $R_{\mathrm{p}}[\mathrm{nm}]$ & $\Delta R_{\mathrm{p}}[\mathrm{nm}]$ \\
\hline \multicolumn{4}{c}{$80 \mathrm{keV} \mathrm{C}^{+}$into $\mathrm{Si}(100)$} \\
\hline Crystal-TRIM & 250 & 226 & 64 \\
ERDA & 243 & 245 & 85 \\
RBS & 260 & 260 & 76 \\
\hline \multicolumn{4}{c}{$40 \mathrm{keV} \mathrm{C}^{+}$into Si $(100)$ at $400^{\circ} \mathrm{C}$} \\
\hline Crystal-TRIM & 120 & 116 & 42 \\
ERDA & 131 & 127 & 55 \\
RBS & 150 & 150 & 59 \\
\hline
\end{tabular}

Depth $_{\max }$ : the depth corresponding to $\mathrm{C}_{\max }$,

$\mathrm{R}_{\mathrm{p}}$ : the projected range,

$\Delta R_{\mathrm{p}}$ : the projected range straggling.

According to Table I, it is clear that Crystal-TRIM code is an accurate program for ion implantation prediction. Indeed, the simulated results (corresponding to $R_{\mathrm{p}}, \Delta R_{\mathrm{p}}$ and depth $\left._{\max }\right)$ are in good agreement with the experiment [8]. Moreover, it is clear that elastic recoil detection analysis (ERDA) measurements are closer to Crystal-TRIM results than the Rutherford backscattering spectroscopy (RBS) data. This is due to the characteristics of the two methods. Indeed, the depth resolution for ERDA technique is reported to be about 10 to $20 \mathrm{~nm}$ [9], whereas for RBS analysis, the depth resolution is reported to be generally around $63 \mathrm{~nm}$ [2]. This means that ERDA measurements are more accurate than $\mathrm{RBS}$.

\section{Conclusion}

In this work, we have performed a simulation study of several parameters corresponding to carbon ion implan- 
tation into silicon targets. It was found that the values of mean projected range $\left(R_{\mathrm{p}}\right)$ and straggling $\left(\Delta R_{\mathrm{p}}\right)$ depended on implantation energies and substrates orientation. Moreover, these values were not influenced by implantation dose and substrate temperature. Furthermore, the shape of carbon depth profiles was a Gaussianlike distribution with the exception of $0^{\circ}$ tilt (i.e. ions beam perpendicular to substrate) which was due to channelling effect. The comparison between our simulated results and those provided in literature confirmed the accuracy of the Crystal-TRIM code.

\section{Acknowledgments}

The authors would like to thank Dr. M. Posselt, from the Institute of Ion Beam Physics and Materials (Germany), for providing the Crystal-TRIM program.

\section{References}

[1] H. Morkoc, S. Strite, G.B. Gao, M.E. Lin, M. Burns, J. Appl. Phys. 76, 1363 (1994).

[2] M. Posselt I, Nucl. Instrum. Methods Phys. Res. B 90, 373 (1994).
[3] M. Posselt, Radiat. Eff. Def. Solids 130-131, 87 (1994).

[4] J.F. Ziegler, J.P. Biersack, U. Littmark, The Stopping and Range of Ions in Solids, Pergamon Press, New York 1985

[5] P. Sigmund, Rad. Efl. 14, 114 (1969).

[6] M. Posselt, Nucl. Instrum. Methods Phys. Res. B 102, 167 (1995).

[7] H. Boudhov, J.P. de Souza, Nucl. Instrum. Methods Phys. Res. B 122, 293 (1997).

[8] S. Intarasiri, T. Kamwanna, A. Hallen, L.D. Yu, M.S. Janson, C. Thongleumd, G. Possnert, S. Singkarat, Nucl. Instrum. Methods Phys. Res. B 249, 859 (2006).

[9] ERDA, Haute Ecole Arc Ingénierie, Neuchâtel 1999. 